\title{
Que peut-on attendre des pratiques d'élevage pour la viande de porcs et autres monogastriques?
}

Jacques MOUROT

Inra,

UMR 1079 SENAH,

35000 Rennes,

France

$<$ <acques.Mourot@rennes.inra.fr>

\begin{abstract}
The monogastric meat of today differs from that produced by the animals 50 years ago. The selection of animals according to growth performance has modified the sensorial and nutritional qualities of the meat. The carcass is less fat and the animals are slaughtered younger at the same weight. The lipid content of the meat is thus decreased. However, in the medical world, meat still has a negative image of being a fat meat whereas this is no longer the case. The rearing conditions influence the quality of monogastrics meat. The feeding level and the fat matter in the diet determine the rate and composition of growth (lipids/proteins) and influence the fatty acids composition. The lipid composition of tissues highly depends on the lipid composition of the diet. Therefore, the fatty acid profile of meat ( $n-3$ fatty acid content for example) can be easily modified through feeding, thereby improving the meat quality for the consumer and meeting the nutritionist's requirements.
\end{abstract}

Key words: meat quality, pig, poultry, rabbit, lipids, fatty acids

et gras) et les produits transformés ont donc une teneur en lipides inférieure à celle des animaux d'après guerre. Et pourtant, la viande continue d'avoir une image négative auprès du monde médical et de certains consommateurs, et elle est accusée d'apporter beaucoup de lipides et d'acides gras saturés (AGS).

Concernant la fraction lipidique de la viande, la qualité nutritionnelle dépend de la quantité de lipide et de la qualité des acides gras (AG). Elle est fortement influencée par les facteurs d'élevage. Ceux-ci sont la génétique, I'alimentation, les conduites et les conditions d'élevage (bâtiment vs plein air, etc.) et des paramètres physiologiques comme l'âge d'abattage et le sexe. Chez les animaux monogastriques, la race, les conditions d'élevage et les paramètres physiologiques vont influencer essentiellement la teneur en lipides de la viande, alors que I'alimentation aura un impact très fort sur la qualité des AG déposés. En effet, il existe une relation forte entre la nature des AG apportés par l'aliment et ceux qui seront présents dans la viande (Wood et al., 2008). Cela est particulièrement vrai chez les animaux monogastriques et à un degré moindre chez les ruminants où le rumen peut transformer jusqu'à $80 \%$ des acides gras poly-insaturés (AGPI) (Chilliard et al., 2008).

Ainsi, il est possible d'utiliser cette relation pour introduire dans I'alimentation animale des AG jugés bons pour la santé humaine et les retrouver dans l'assiette du consommateur (Chilliard et al., 2008). Cette notion d'AG jugés bons pour la santé peut varier avec le temps. II y a plus de 20 ans, un des objectifs en production animale monogastrique était d'accroître la teneur en acide linoléique (C18:2 n-6-LA), alors que la tendance actuelle est de s'intéresser davantage aux AG de la famille n-3. Cet objectif correspond à un souhait de l'Afssa qui propose d'augmenter la part du précurseur C18:3 n-3 (acide à linolénique-ALA) dans I'alimentation humaine et de diminuer celle du C18:2 n-6 (ANC, 2001). L'objectif global est d'arriver à un rapport LA/ALA voisin de 5, alors qu'il est actuellement estimé entre 15 et 30 . Pour l'Afssa, tout vecteur alimentaire qui contribuera à apporter le précurseur ALA dans I'alimentation humaine est à prendre en considération. La viande et les produits animaux peuvent largement y contribuer, car ils représentent déjà plus de $50 \%$ de cet apport (Boue et Combe, 2001). Concernant les AG des viandes issues de production standard, il apparaît que les AG majoritaires sont les mono-insaturés (dont plus de $40 \%$ d'acide oléique) chez le porc et la volaille, alors que chez le lapin les AGS sont majoritaires, mais chez cette espèce, les trois familles saturées, mono-insaturées et poly-insaturées sont relativement équivalentes (tableau 1). D'une manière générale, il est donc faux de continuer de dire que les viandes sont surtout des sources importantes d'AGS, ce n'est plus le cas avec les viandes d'aujourd'hui.

\section{Évolution de la composition de la viande de porc}

Tout d'abord, il convient de rappeler que la viande de porc est une viande maigre. En effet, lorsqu'elle est dans l'assiette du consom- 
Tableau 1. Composition moyenne en acides gras (\%) en fonction des espèces et des sites anatomiques (muscles: longissimus dorsi pour le porc et le lapin, pectoralis pour le poulet, tissus adipeux : sous-cutané du dos pour le porc, abdominal pour le poulet et interscapulaire pour le lapin) [synthèse de données de notre laboratoire; $\mathrm{n}=8$ à 12 animaux par régime].

\begin{tabular}{|c|c|c|c|c|c|c|}
\hline & \multicolumn{3}{|c|}{ Muscle } & \multicolumn{3}{|c|}{ Tissu adipeux } \\
\hline & Porc & Poulet & Lapin & Porc & Poulet & Lapin \\
\hline AGS & 37,5 & 33,2 & 39,8 & 39,1 & 33,4 & 40,6 \\
\hline AGM & 45,3 & 46,5 & 30,0 & 45,7 & 48,2 & 34,4 \\
\hline AGPI & 17,2 & 20,3 & 30,2 & 15,2 & 17,4 & 25,0 \\
\hline C16:0 & 24,4 & 25,6 & 28,4 & 24,1 & 25,6 & 31,4 \\
\hline C18:0 & 11,5 & 6,4 & 8,0 & 13,4 & 7,4 & 5,8 \\
\hline C18:1 & 40,8 & 40,3 & 25,9 & 42,5 & 43,3 & 30,1 \\
\hline C18:2 n-6 & 12,7 & 14,0 & 21,2 & 12,9 & 14,5 & 21,2 \\
\hline C18:3 n-3 & 0,4 & 1,1 & 1,5 & 1,0 & 1,0 & 3,3 \\
\hline LA/ALA & 30,6 & 12,4 & 14,1 & 12,8 & 14,6 & 6,4 \\
\hline
\end{tabular}

AGS : acides gras saturés ; AGM : AG mono-insaturés ; AGPI : AG poly-insaturés. mateur, ce dernier peut écarter facilement le gras visible. De nombreuses études ont montré muscle principal du rôti de porc) lorsqu'il est paré, contient moins de $2 \%$ de lipides. Comme pour toutes les espèces animales, la teneur en lipides dépendra de la localisation anatomique. Pour la côte de porc entière, la teneur en lipides est voisine de 10 à $12 \%$, mais déjà au niveau de l'étal, le boucher peut choisir de diminuer l'épaisseur du gras dorsal, et dans ce cas, la teneur en lipides peut alors être inférieure à $5 \%$ (Mourot et Lebret, 2009). II ne faut pas oublier non plus que la viande est aussi une source importante de protéines (17 à $20 \%$ selon les morceaux et les espèces), de minéraux et de certaines vitamines (Culioli et al., 2003), donc proscrire la consommation de viande pour diminuer la part des lipides dans I'alimentation humaine est trop restrictif.

Comme il a été rappelé précédemment, la nature des matières grasses de l'aliment va influencer la composition en AG de la viande. Par exemple, l'introduction d'acide oléique soit par I'utilisation de I'huile d'olive dans l'aliment (Nuernberg et al., 2005) ou de tournesol oléique (Albar et al., 2000) permet d'augmenter notablement le dépôt d'acide oléique de plus de dix points et de diminuer celui des AG n-6.

De nombreuses études sont menées actuellement pour accroître la teneur en AG n-3 de la viande en apportant dans l'alimentation animale du lin ou du colza et plus récemment du chanvre. II faut aussi savoir que la teneur en lipides du régime du porc est relativement faible (3 à $4 \%$ en pondéral), il n'y a donc pas de doses massives de matière grasse incorporées dans l'aliment. que le muscle longissimus dorsi, (c'est-à-dire le
Des résultats contradictoires apparaissent avec le lin. Cela est la conséquence des variétés de lin utilisées dont plus de 190 variétés existent dans la nature (Rousseau, 2005). Il existe des graines de lin pauvres en matière grasse $(10 \%)$ et en AG n-3 (10 à $15 \%$ des AG totaux) et des graines de lin sélectionnées pour leur teneur en matière grasse $(50 \%)$ et riches en AG $n-3$ (près de $60 \%$ des AG). Plus concrètement, cet écart peut aller de 1 à 25 pour la teneur en ALA dans la graine de lin. Les plus riches en ALA seront donc plus efficaces, car elles apporteront davantage d'AG n-3. L'introduction de graines de lin extrudées dans I'aliment du porc apparaît également plus efficace que I'huile de lin ou des graines toastées pour obtenir un meilleur dépôt $\mathrm{d}^{\prime} \mathrm{AG} \mathrm{n}-3$ dans la viande en raison d'une meilleure digestibilité (Noblet et al., 2008).
Des études réalisées chez le porc en croissance et le porc lourd ont montré que l'on peut augmenter fortement la teneur en AG C18:3 dans la viande d'animaux recevant des graines de lin extrudées riches en C18:3 (Corino et al., 2008). Globalement, pour des régimes isolipidiques, la quantité de ALA et de $n-3$ retrouvés dans la viande est en relation avec la quantité de précurseur n-3 ingérée (tableau 2). Cependant, les $A G$ à longue chaîne, dérivés du précurseur DHA (CC22:6 n-3), sont peu augmentés. Cela est la conséquence d'une faible activité des delta 5 et 6 désaturases comme observé chez la plupart des animaux et chez l'Homme (Alessandri et al., 2009).

Le rapport LA/ALA varie de 29 (avec I'huile de tournesol source de n-6) à 2 avec le lin. Avec les matières grasses apportées par le colza, le chanvre ou le lin, ce rapport est conforme aux souhaits de l'Afssa (ANC, 2001) avec une valeur proche ou inférieure à 5 , mais c'est avec le lin et plus particulièrement avec les graines extrudées que la teneur en ALA de la viande sera la plus élevée. Cette viande peut alors revendiquer une allégation riche en ALA, puisque que la teneur représente $15 \%$ des besoins journaliers. Si l'on considère que le poids moyen d'une côte de porc représente souvent $150 \mathrm{~g}$ de viande, l'apport sera alors d'environ de $450 \mathrm{mg}$ d'ALA, ce qui fait une augmentation de plus de $360 \mathrm{mg}$ d'ALA dans I'alimentation humaine par rapport à un porc standard. Sachant que le déficit de consommation d'ALA est estimé par la plupart des enquêtes alimentaires aux environs de $1,1 \mathrm{~g}$ par jour, la côte de porc issue d'animaux recevant du lin permettra de combler à elle seule un tiers de ce déficit.

La transformation n'altère pas la teneur en ALA des produits ni leur qualité sensorielle. Globalement, la teneur sera multipliée par 4 à 7 selon la C18:3 n-3 comme l'acide EPA (C20:5 n-3) et

Tableau 2. Relation entre les quantités de LA et ALA ingérées et celles déposées dans la côte de porc en fonction de la nature de la matière grasse de l'aliment (synthèse de données de notre laboratoire; $\mathrm{n}=8$ à 12 porcs par régime).

\begin{tabular}{|c|c|c|c|c|c|c|c|c|c|}
\hline \multirow[t]{2}{*}{ Matière grasse } & \multicolumn{2}{|c|}{$\begin{array}{l}\text { AG ingéré } \\
\mathrm{g} / \mathrm{kg} \text { aliment }\end{array}$} & \multicolumn{7}{|c|}{ Milligrammes d'AG par $100 \mathrm{~g}$ de côte de porc crue } \\
\hline & $\overline{\text { LA }}$ & ALA & $\overline{\text { LA }}$ & ALA & EPA & DPA & DHA & S n-3 & LA/ALA \\
\hline Palme & 10,19 & 0,61 & 875 & 63 & 5,5 & 10,6 & 4,3 & 84 & 17 \\
\hline Tournesol & 19,18 & 0,67 & 1264 & 52 & 4,0 & 8,4 & 3,6 & 68 & 29 \\
\hline Soja & 13,63 & 1,76 & 1151 & 96 & 5,8 & 14,3 & 4,0 & 120 & 8 \\
\hline Colza & 12,81 & 2,02 & 898 & 106 & 4,8 & 12,2 & 3,6 & 127 & 6 \\
\hline Chanvre & 17,21 & 3,43 & 1243 & 161 & 6,7 & 16,8 & 4,3 & 189 & 5 \\
\hline Huile de lin & 11,39 & 5,19 & 949 & 274 & 10,8 & 22,2 & 4,3 & 311 & 2 \\
\hline $\begin{array}{l}\text { Graines de lin } \\
\text { extrudées }\end{array}$ & 10,51 & 5,75 & 869 & 300 & 13,8 & 24,5 & 5,5 & 344 & 2 \\
\hline
\end{tabular}


teneur en lipides des produits parmi des animaux recevant du lin par rapport à une matière grasse standard (souvent du palme en ce moment pour des raisons économiques). De plus, cette augmentation en quantité d'AG $\mathrm{n}-3$ est souvent conjointe à une diminution de la fraction des AGS (Guillevic et al., 2009).

Il faut toutefois noter que des produits de charcuterie sèche peuvent présenter des défauts d'odeur en raison d'une oxydation des AG (Musella et al., 2009 ; Cannata et al., 2010), mais dans ces essais les quantités d'AG n-3 de I'aliment étaient supérieures à celles usuellement recommandées en élevage ; la quantité dans le jambon sec était donc plus élevée, ce qui accroît la susceptibilité à l'oxydation. Mais l'ajout d'antioxydants dans I'aliment comme la vitamine $E$ couplée à des antioxydants végétaux permet de diminuer ces risques d'oxydation, ce qui conduit à des produits sans défaut d'odeur et jugés bons par le consommateur (Mairesse et al., 2010).

L'introduction, dans I'aliment du porc, de DHA (apporté par des algues) en grande quantité ne semble pas donner les résultats attendus

Tableau 3. Teneur en lipides et composition en AG de différents tissus chez des poulets recevant un régime standard et abattus à 42 jours (Ratnayake et al., 1989).

\begin{tabular}{|lrcr|}
\hline & Filet & Cuisse & Peau \\
\hline Lipides (\% du tissu frais) & 0,9 & 2,3 & 26,9 \\
\hline AG (\% des AG totaux) & & & \\
\hline C16:0 & 23,8 & 22,6 & 24,0 \\
\hline C18:0 & 7,5 & 7,6 & 5,1 \\
\hline C16:1 & 4,5 & 6,3 & 7,8 \\
\hline C18:1 & 29,1 & 32,0 & 39,4 \\
\hline C20:1 & 0,5 & 0,5 & 0,6 \\
\hline C22:1 & 0,4 & 0,6 & 0,4 \\
\hline C18:2 n-6-LA & 17,8 & 18,3 & 18,2 \\
\hline C20:4 n-6 & 5,0 & 3,7 & 0,6 \\
\hline C18:3 n-3-ALA & 0,5 & 0,7 & 1,0 \\
\hline C20:5 n-3 & 0,7 & 0,6 & 0,4 \\
\hline C22:5 n-3 & 0,9 & 0,5 & 0,1 \\
\hline C22:6 n-3 & 1,8 & 1,0 & 0,1 \\
\hline Total AGPI-LC & 8,4 & 5,8 & 1,2 \\
\hline Total AGPI-LC n-3 & 3,4 & 2,1 & 0,6 \\
\hline LA/ALA & 35,6 & 26,1 & 18,2 \\
\hline ALA (mg/100 g de tissu) & 3,1 & 13,3 & 242 \\
\hline DHA (mg/100 g de tissu) & 11 & 19 & 24 \\
\hline
\end{tabular}

comme avec ALA. La viande est certes enrichie en cet $A G$, mais pas autant que I'on pouvait espérer. Un apport de $2 \mathrm{~g}$ de DHA/kg d'aliment contre 0,02 g pour l'aliment standard (soit un écart de 100) permet d'obtenir une viande enrichie en DHA entre six et dix fois la valeur basale, soit une valeur de 50 à $70 \mathrm{mg}$ pour $100 \mathrm{~g}$ de viande. Cet AG est vraisemblablement utilisé pour l'oxydation peroxysomale, et il est donc peu disponible pour le stockage (Mourot, 2009). Des études sont en cours pour essayer d'augmenter le dépôt de cet AG. Une durée courte de distribution (environ trois semaines) avant que la $\beta$-oxydation peroxisomale ne soit adaptée à cet $\mathrm{AG}$ où l'apport conjoint du DHA et de son précurseur ALA semblent des pistes intéressantes.

\section{Évolution de la composition de la viande de volaille}

La teneur en lipides de la carcasse des volailles varie en fonction de la souche, des conduites alimentaires et des systèmes d'élevage (plein air vs bâtiment) (Lessire, 2001). La carcasse de dinde est la plus maigre avec environ de 7 à $10 \%$ de lipides. Chez le poulet standard, abattu à 42 jours, la teneur en lipides atteint environ $18 \%$, et le canard non gavé contient un peu plus de lipides avec une valeur proche de $20 \%$. Ces lipides ne sont pas répartis uniformément dans la carcasse ; une partie non négli- geable se trouve dans la cavité abdominale (gras abdominal et gras de viscères) et en périphérie de la carcasse (gras sous-cutané). Le gras abdominal représente jusqu'à $4 \%$ du poids vif chez les animaux modernes; il renferme environ $88 \%$ de lipides. Une carcasse prête à cuire (PAC) est débarrassée à l'abattoir de ce gras abdominal ainsi que des viscères (intestin, cœur, poumons, etc.), elle est donc moins riche en lipides.

La quantité de lipides varie également selon les tissus. Les filets de poulet sont plus maigres $(0,9 \%)$ que la cuisse $(2,8 \%)$, et la peau est nettement plus grasse : environ $27 \%$ (Ratnayake et al., 1989). La commercialisation de la volaille sous forme de pièce de découpe tend à se développer. Dans ce cas, le filet est proposé aux consommateurs majoritairement sans la peau et apporte donc peu de lipides, alors que les cuisses sont commercialisées entières avec la peau. Chez la dinde, les muscles de la cuisse sont également plus riches en lipides que le filet. Chez le canard, ces deux masses musculaires ont des teneurs similaires en lipides (2\%).

La composition en AG des tissus de poulets recevant un régime standard figure au tableau 3 (Ratnayake et al., 1989). Cent grammes de muscle rouge (la cuisse) contient deux fois plus de matières grasses que le muscle blanc (le filet), et ils apportent cinq fois plus d'ALA (14 mg /100 g vs $3 \mathrm{mg}$ ).

Une étude récente montre (figure 1) que la teneur en ALA de la carcasse est pratiquement

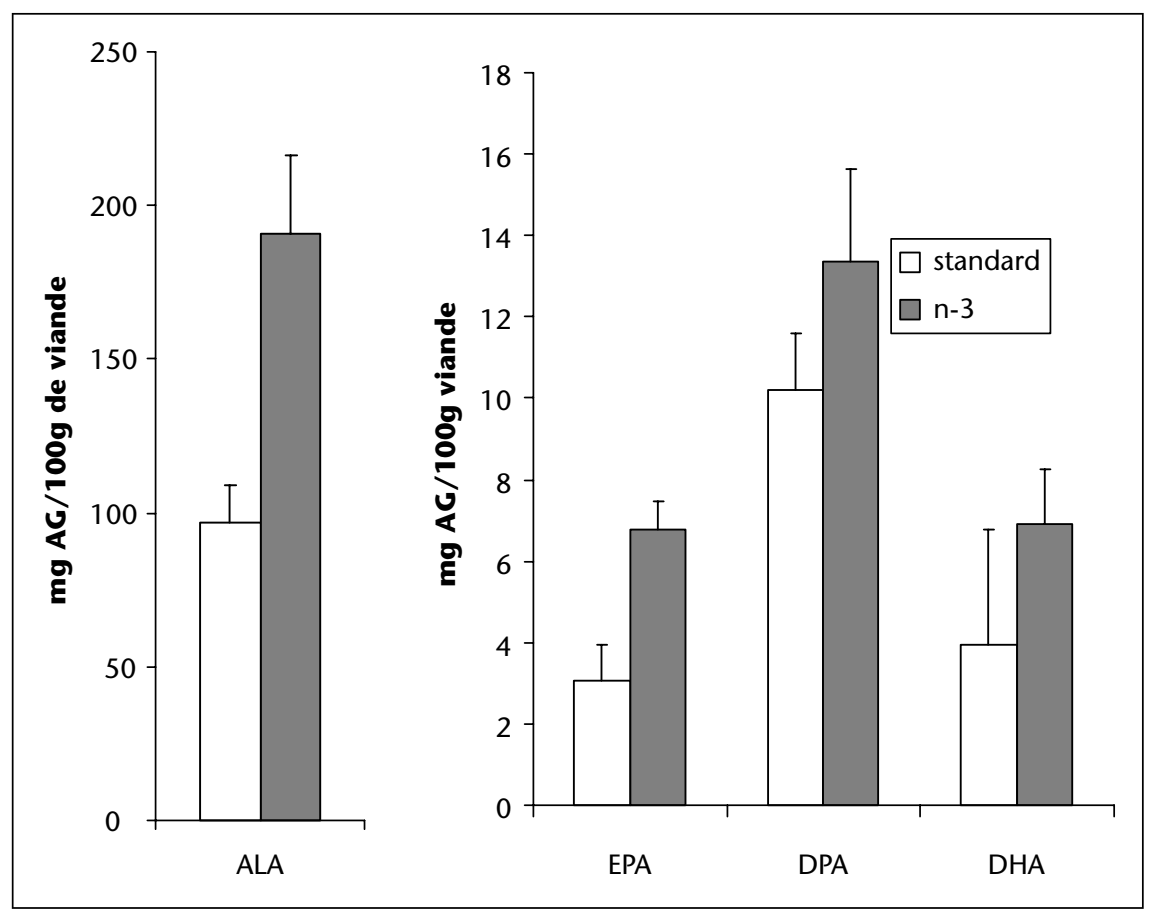

Figure 1. Effet de l'apport de graines de lin extrudées sur la teneur en ALA et en dérivés de la carcasse (en milligrammes d'AG par $100 \mathrm{~g}$ de viande) [régime témoin contenant $0,74 \mathrm{~g}$ d'ALA/ $\mathrm{kg}$ de régime et régime $n-3$ avec $1,9 \mathrm{~g} / \mathrm{kg}$ ] (Mourot données non publiées). 
doublée $(p<0,001)$ alors que la teneur dans I'aliment est multipliée par 2,6. La teneur en EPA est également doublée mais, la valeur basale étant très faible, l'accroissement en d'EPA est donc négligeable. Il en est de même pour le DPA et le DHA. Mais, globalement, la teneur en acides gras n-3 est faible par rapport aux autres espèces monogastriques, car la teneur en lipides de la volaille est faible.

\section{Évolution de la composition de la viande de lapin}

Traditionnellement, la viande de lapin jouit d'une bonne image au point de vue de sa qualité nutritionnelle (Combes, 2004). Elle a une teneur moyenne en lipides qui varie de 8 à $13 \%$ en fonction des conditions de production. Il est généralement admis que, pour des conditions d'élevage similaires et à un même âge, les lapins les plus lourds sont les plus gras. Comme pour la plupart des espèces, la viande de lapin contient de 0,5 à $1 \mathrm{~g}$ de phospholipides pour $100 \mathrm{~g}$ de tissu frais (Gondret, 1998), et les triglycérides de leur côté représentent environ 85 à $90 \%$ des lipides de la viande. Contrairement au porc qui présente un tissu adipeux de couverture sur l'ensemble de la carcasse, les masses adipeuses visibles chez le lapin sont le gras interscapulaire (entre les épaules) et surtout le gras abdominal. Un dégraissage manuel de la carcasse par enlèvement de ces deux masses adipeuses visibles avant la cuisson réduit la teneur moyenne en lipides d'une carcasse de 12,5 à 10,7 \% (Gigaud et Le Cren, 2006).

Les morceaux de découpe commerciale ont une teneur variable en lipides en fonction de leur position anatomique : les pattes avant et le râble sont les parties les plus grasses, et les cuisses sont les parties les plus maigres (Ouhayoun et Delmas, 1989).

Traditionnellement, l'aliment du lapin contient une part non négligeable de luzerne sources de protéines mais aussi de fibres. La proportion courante de luzerne dans les rations est de l'ordre de 15 à $20 \%$, mais cette proportion peut sans aucune contre-indication être augmentée jusqu'à 55-60\%. Toutefois, pour des raisons économiques, la part de luzerne tend à diminuer actuellement et est remplacée en partie par des tourteaux de tournesol.

Le tableau 4 rapporte la teneur moyenne en AG de différents morceaux de carcasse de lapin recevant un aliment standard (Kouba et al., 2008).

Les caractéristiques nutritionnelles de la viande de lapin comme celle des autres animaux monogastriques sont influencées par la nature des matières grasses de leur aliment.

Des essais récents apportant plus ou moins de teneur en ALA dans I'aliment via des graines de lin extrudées ont été réalisés. Les valeurs moyennes de teneur en ALA déposées dans la viande en fonction de la teneur dans l'aliment sont rapportées dans la figure 2 .

Cet accroissement de la teneur en ALA, et plus globalement en AG n-3, se fait principalement au détriment des teneurs en $L A$. Cela permet de diminuer le rapport LA/ALA, ce qui va dans le sens des recommandations de l'Afssa. II apparaît également que la teneur en AGS peut être diminuée.

Les teneurs en EPA et en DHA sont très faibles chez le lapin, ces AG varient peu avec l'introduction du précurseur ALA. Des études ont semblé montrer que ces AGPI-LC n-3 pouvaient augmenter avec l'apport de graines de lin extrudées dans l'aliment, mais l'apport d'huile de poisson comme source basale de matière grasse n'avait pas été pris en considération dans l'interprétation des résultats, ce qui a certainement faussé les conclusions. II semblerait donc que le lapin désature encore plus mal le précurseur ALA que les autres animaux monogastriques.

Les pièces de découpe (à l'exception de la cuisse) issues de lapin ayant ingéré une quantité suffisante de précurseurs n-3 peuvent prétendre à l'allégation source de $n-3$. Mais la faible part de viande de lapin consommée, moins de $2 \mathrm{~kg}$ par an et par habitant, contribuera peu à accroître l'apport de n-3 dans l'alimentation humaine.

Tableau 4. Teneur moyenne en lipides totaux ( $\mathrm{g} / 100 \mathrm{~g}$ de viande) et en acides gras $(\mathrm{mg} / 100 \mathrm{~g})$ dans la viande de lapin standard, comparaison entre les morceaux de découpe (d'après Kouba et al., 2008).

\begin{tabular}{|c|c|c|c|c|c|}
\hline & Carcasse & Épaule & Cuisse & Râble & Foie \\
\hline Lipides totaux & 8,37 & 7,81 & 4,37 & 8,99 & 3,99 \\
\hline $\mathrm{C} 14: 0$ & 197 & 114 & 60 & 134 & 15 \\
\hline C14:1 & 31,54 & 6,7 & 4,5 & 7,6 & 5,9 \\
\hline $\mathrm{C} 16: 0$ & 1657 & 1531 & 844 & 1795 & 304 \\
\hline $\mathrm{C} 16: 1(\mathrm{n} 7)$ & 156 & 157 & 88 & 187 & 38 \\
\hline C18:0 & 432 & 282 & 166 & 331 & 224 \\
\hline C18:1(n9) & 1648 & 1461 & 793 & 1717 & 285 \\
\hline C18:2(n6) & 1533 & 1052 & 599 & 1224 & 311 \\
\hline$C 20: 0$ & 1,74 & 7,0 & 4,1 & 8,4 & 2,6 \\
\hline $\mathrm{C} 18: 3(\mathrm{n} 3)$ & 143 & 152 & 80 & 178 & 21 \\
\hline $\mathrm{C} 20: 1(\mathrm{n} 9)$ & 13,10 & 18,6 & 10,2 & 22,2 & 5,8 \\
\hline $\mathrm{C} 20: 2$ & 4,14 & 8,8 & 5,4 & 10,2 & 4,3 \\
\hline $\mathrm{C} 20: 3(\mathrm{n} 3)$ & 10,85 & 5,6 & 4,9 & 6,5 & 11,5 \\
\hline$C 20: 4(n 6)$ & 1,91 & 28 & 29 & 29 & 81 \\
\hline $\mathrm{C} 22: 1(\mathrm{n} 9)$ & 0,70 & 2,2 & 1,7 & 2,4 & 1,6 \\
\hline $\mathrm{C} 20: 5(\mathrm{n} 3)$ & 3,32 & 3,0 & 2,4 & 3,3 & 6,2 \\
\hline C24:0 & 9,60 & 8,3 & 8,1 & 8,8 & 9,8 \\
\hline $\mathrm{C} 24: 1(\mathrm{n} 9)$ & 18,53 & 4,8 & 3,9 & 5,0 & 8,0 \\
\hline $\mathrm{C} 22: 5(\mathrm{n} 3)$ & 9,20 & 6,2 & 5,7 & 6,6 & 6,9 \\
\hline $\mathrm{C} 22: 6(\mathrm{n} 3)$ & 1,90 & 3,9 & 2,5 & 4,5 & 3,7 \\
\hline AGS & 2355 & 1942 & 1083 & 2277 & 515 \\
\hline AGM & 1869 & 1651 & 901 & 1942 & 319 \\
\hline AGPI & 1708 & 1260 & 729 & 1461 & 413 \\
\hline$n-6$ & 1546 & 1080 & 628 & 1253 & 364 \\
\hline$n-3$ & 158 & 171 & 95 & 198 & 45 \\
\hline LA/ALA & 10,66 & 6,91 & 7,49 & 6,89 & 15,12 \\
\hline
\end{tabular}




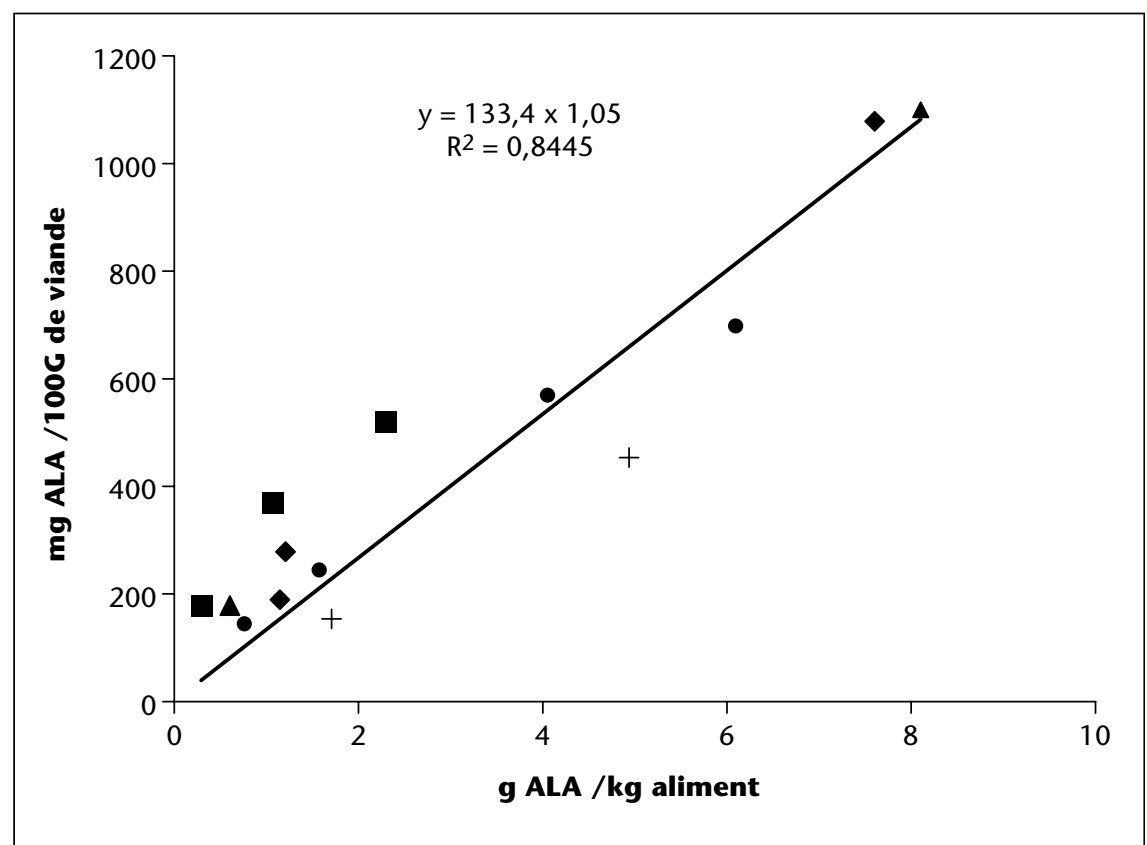

Figure 2. Évolution de la teneur moyenne de la viande de lapin en ALA en fonction de la teneur en cet AG dans l'alimentation des lapins en engraissement. D’après Combes et Cauquil, 2006 ; Gigaud et Le Cren, 2006; Colin et al., 2005 ; Kouba et al., 2008 ; Mourot, 2009 (en cours de publication).

\section{Conclusion}

Les pratiques d'élevage peuvent modifier la qualité nutritionnelle des produits animaux et plus particulièrement des monogastriques. Il est indéniable que ces animaux sont maintenant moins gras que ceux que l'on produisait il y a une cinquantaine d'années. Cela est donc particulièrement intéressant pour la santé humaine, car l'apport de lipides a diminué dans l'assiette du consommateur. L'introduction d'AG n-3 dans I'aliment de l'animal permettra de les retrouver dans l'assiette du consommateur, mais globalement, c'est essentiellement le précurseur $\mathrm{C} 18: 3 \mathrm{n}-3$ qui sera retrouvé dans la viande, la transformation en AGPI à longues chaines $n-3$ restera limitée. Ces apports de $n-3$ permettront de rééquilibrer le rapport n-6/n-3 dans la viande des animaux monogastriques, ce rapport passant de 10 à 15 à une valeur inférieure ou voisine de 5. Sachant que les viandes de porc et de volailles sont très consommées, ces pratiques d'élevage peuvent donc permettre d'apporter davantage d'AG n-3 dans I'alimentation humaine et participer à rééquilibrer l'apport d'AG n-6/n-3.

Maintenant, il est nécessaire que les spécialistes de la nutrition humaine revoient leur jugement négatif vis-à-vis de la consommation des viandes et des produits animaux en général. Enfin, puisque l'alimentation des animaux peut modifier la composition en AG des viandes, il faut renforcer les échanges
Colin M, Raguenes N, Le Berre G, Charrier S, Priogent AY, Perrin G. Influence d'un enrichissement de I'aliment en acides oméga-3 provenant de graines de lin extrudées (Tradi-Lin ${ }^{\circledR}$ ) sur les lipides et les caractéristiques hédoniques de la viande de lapin. $11^{e}$ Journées de la recherche cunicole. Paris, 2005: 163-6.

Combes $S$. Valeur nutritionnelle de la viande de lapin. INRA Prod Anim $2004 ; 17$ : 373-83.

Combes S, Cauquil L. Viande de lapin et oméga-3 : une alimentation riche en luzerne permet d'enrichir la viande des lapins en oméga-3. Viande \& Produits Carnés 2006 ; $25: 31-5$.

Corino C, Musella M, Mourot J. Influence of extruded linseed on growth, carcass composition and meat quality of pigs slaughtered at 110 and $160 \mathrm{~kg}$ liveweight. / Anim Sci 2008 ; 86 : 1850-60.

Culioli J, Berri C, Mourot J. Muscle foods: consumption, composition and quality. Sci Aliments 2003; $23: 13-34$.

Gigaud V, Le Cren D. Valeur nutritionnelle de la viande de lapin et influence du régime alimentaire sur la composition en AG. Journée nationale ITAVI élevage du lapin de chair. Pacé 2006 : 45-57.

Gondret F. Lipides intramusculaires et qualité de la viande de lapin. $7^{e}$ Journée. Paris, France : Rech. Cunicole, 1998.

Guillevic M, Kouba M, Mourot J. Effect of a linseed diet on lipid composition, lipid peroxidation and consumers evaluation of fresh meat and French cooked pork meats. Meat Sci $2009 ; 81: 612-8$.

Kouba M, Benatmane F, Blochet JE, Mourot J. Effect of a linseed diet on lipid oxidation, fatty acid composition of muscle, perirenal fat, and raw and cooked rabbit meat. Meat Sci 2008; 80 : 829-34.

Labroue F, Marsac H, Luquet M, et al. Performances of French local breeds. In: Pig genetic resources in Europe. EAAP publication $n^{00} 104$. Wageningen Pers, $2001: 51-7$.

Lessire M. Matières grasses alimentaires et composition lipidique des volailles. INRA Prod Anim 2001 ; 14 : 365-70.

Mairesse G, Benet M, Meteau K, Juin H, Durand D, Mourot J. L'ajout d'antioxydants végétaux dans l'alimentation des porcs permet-il de limiter la peroxydation des viandes enrichies en acides gras polyinsaturés n-3? Journées de la Recherche Porcine $2010 ; 42$ (sous presse).

Mourot J. Du contrôle de la masse adipeuse chez les animaux de rente. Bull Acad Vet Fr $2004 ; 157$ : 29-34.

Cannata S, Ratti S, Meteau K, Mourot J, Baldini P, Corino $C$. Evaluation of different types of dry-cured ham by Italian and French consumers. Meat Sci 2010 ; 84 : 601-6.

Chilliard Y, Bauchart D, Lessire M, Schmidely P, Mourot J. Qualité des produits : modulation par I'alimentation des animaux de la composition en acides gras du lait et de la viande. INRA Prod Anim 2008 ; 21 : 95-106.
Mourot J. Optimising the nutritional and sensorial profile of pork. In: Kerry JP, Ledward DA, eds. Improving the sensory and nutritional quality of fresh meat. In food science technology and nutrition. England: Woodhead Publishing Cambridge, CB1 6AH, 2009, 342-55. 
Mourot J, Lebret B. Modulation de la qualité de la viande de porc par I'alimentation. INRA Prod Anim $2009 ; 22$ : 33-40.

Musella M, Cannata S, Rossi R, Mourot J, Baldini P, Corino C. Influence of n-3 PUFA from extruded linseed on fresh and dry-cured ham quality of slaughtered pigs at $160 \mathrm{~kg}$ liveweight: n-3 PUFA from extruded linseed influences fatty acid composition and sensory characteristics of dry-cured ham from heavy pigs. I Anim Sci 2009; 87 : 3578-88.
Noblet ], Jaguelin-Peyraud Y, Quemeneur B, Chesneau G. Valeur énergétique de la graine de lin chez le porc : impact de la technologie de cuisson-extrusion. Journées de Recherche Porcine 2008 ; 40 : 203-8.

Nuernberg K, Fischer K, Nuernberg G, et al. Effects of dietary olive and linseed oil on lipid composition, meat quality, sensory characteristics and muscle structure in pigs. Meat Sci $2005 ; 70: 63-74$.

Ouhayoun J, Delmas D. La viande de lapin : composition de la fraction comestible de la carcasse et des morceaux de découpe. Cuni-Sciences $1989 ; 5$ : 1-6.
Ratnayake WMN, Ackman RG, Hulan HW. Effect of redfish meal enriched diets on the taste and $n-3$ PUFA of 42-day-old broiler chickens. / Sci Food Agric $1989 ; 49: 59-74$.

Rousseaux E. Le lin et ses secrets. La Crèche: Geste Editions, 2005.

Wood JD, Enser M, Fisher AV, et al. Fat deposition, fatty acid composition and meat quality: a review. Meat Sci 2008 ; 78 : 343-58. 